\title{
Efficient Eye Detection and Tracking Using Smart Sensors
}

\author{
Hariharan S, Nagappan G, Vijayanand M, Vidhya Sri S, Sree Ranjhani R S
}

\begin{abstract}
Smart sensing enables applications with lots of computer-based techniques and algorithms to work in a finer way. We are in era with intelligent machines around us and there sometimes provides a situation of spending of large amount of time due to lack of understanding of those devices. In this paper, we attempt on to find a new interaction method which ease the use of computers or increase interaction efficiency. The most interesting approach that enhances the best way of to find communication between machines and human beings. This enhances the way of communication with the digital devices. Our project enables to have communication using our eye in the desktop environment. This study focus on using eye gazing techniques that allows user to move the mouse cursor using his eye with interpretation of control commands. The desktop environment which has the inbuilt camera enables to achieve the goal in simple and efficient way. The study includes analysis of features using face detection and template matching which have presented promising results.
\end{abstract}

Index Terms- Detection, Eye Tracking, Scrolling, Smart sensing, Sensors, Human Computer Interaction.

\section{INTRODUCTION}

Eye gaze tracking offers a range of potential apps including interaction with human-computers, virtual realities and the diagnosis of visual conditions. The application that we focus would help the people who are potentially disabled and they were unable to interact with the computer in a normal manner [6]. Furthermore, those applications were designed to control the mouse pointer from normal human eyes to enable the user to speed up the focus point selection. An added feature would be information integration which would provide improved security as well as ease of the access among the control systems with a standard template [8]. A gaze would be used in studying the human cognition, memory, behavior along with several other factors and are multi faceted in nature with adaptation of standard libraries [9]. The study of human monitoring closely links with the detection of visual sensing revealing the focus of the person. Several approaches exist earlier to accomplish the task of gaze tracking. Such visual tracking techniques utilize these intrusive devices which require physical contact with users. Video-based gaze tracking techniques are instead more suitable for daily use and could offer an effective nonintrusive solution.

Revised Manuscript Received on 14, October 2019.

Hariharan S, Saveetha Engineering College, Chennai, Tamilnadu, India(E-mail: hariharans@ saveetha.ac.in)

Nagappan G, Saveetha Engineering College, Chennai, Tamilnadu, India (E-mail: nagappan.cse@ saveetha.ac.in)

Vijayanand M, Saveetha Engineering College, Chennai, Tamilnadu, India(E-mail: vijayanand@ saveetha.ac.in)

Vidhya Sri S, Saveetha Engineering College, Chennai, Tamilnadu, India(E-mail: vidhyas996@gmail.com)

Sree Ranjhani R S, Saveetha Engineering College, Chennai, Tamilnadu, India(E-mail: sanran1997@gmail.com)
From the study, we found that there are two types of imaging techniques which were commonly utilized in videobased approaches namely infrared and visible imaging. The former type of imaging system uses infrared cameras along with infrared light sources to capture pictures with low grade followed by analysis [7]. The latter usually uses high resolution photo cameras. Infrared imagery uses invisible infrared light. A controlled illumination source normally would reduce the impact on light. Such reduction process normally would reduce the impact of light on pupil creating a strong contrast between iris, pupil and pupil. This enables an infrared image-based method to track the eye gaze. This class includes the majority of video approaches with normal pictures. In this section, we present some remarks based on this aspect as:

$\checkmark \quad$ An infrared system will not be reliable when the other infrared sources are disturbed.

$\checkmark \quad$ All-user system will not produce an light dark effect that could fail the gaze tracker.

$\checkmark \quad$ Infrared light source reflex on the glass remains an issue.

Visible imagery techniques have strong impact based on the above characteristics. There also remain several other problems as compared to pictures extracted using infra red instruments. They are not vulnerable to the use of infrared sources and glasses in the environment. Usually images have controlled behavior with lower contrast and brightness on par with images explored to natural environment with ambient light source which are uncontrolled. The detection of iris center is possibly a hard task than the detection of pupil center. It is basically because of the fact that the upper eyelid is usually partially contained in the iris. In this paper, we mainly focus only on visible imaging methods on par with human and computer interaction and related procedures [3]. The approach to the eye gaze tracking and the study and related wearable environment across different languages exist [1]. There also exists systems for users who are physically challenged and those who needs special needs [5] use these systems in a efficient manner [4].

The paper is organized in the following manner. While section I addressed on basic information, related work is presented in section II. The algorithm work flow process is briefed in Section III followed by experimental analysis in section IV. Section V describes the evaluation metrices and 
final section (section VI) gives the conclusions from the study made and future work.

\section{RELATED WORK}

This section examines some of the related work investigated with visible imaging gaze tracking methods, functions and appearances. The characteristic of gaze tracking mainly depends on the extraction of eye area characteristics with iris center and contour, to provide information on the movement of the eye. There are several studies with the combination of position of the eye and head [11]which are presented in this section in a detailed manner .

Zhu and Yang [10] carried out an image of intensity feature extraction. A preset eye corn filter was used to extract the eye corner and the center of the iris eye was measured by the interpolated size of the Sob ledge. A linear mapping function then determined the direction of the look. Here in the study, users keep their head stable, since the direction of the gaze is sensitive to the head position.

Torricels et al [12] used corner detection methods for obtaining geometrical characteristics that are mapped to the general neural network (GRNN) screen coordinates. The system accuracy is generally heavily dependent on these input vectors and deteriorates with errors in any input vector element.

Ince and Kim [13] have designed on an tracking procedure which involves deformable eye center detection and motion. The decision algorithms strongly depend on the shape and intensity. Its system performed in video sequences with small resolution, but its accuracy was much sensitive to head posture. Evidence based look tracking doesn't extract the features, but instead uses the entire image to estimate the gaze.

Sugano et al [14] introduced the online learning algorithm, which uses the user's operations like mouse click using the design interface within a incremental learning frame. With every mouse click, a sample mouse coordinate was recorded and labeling was done (i.e., head position and eye picture), associated with the features. It was therefore difficult to get a lot of samples.

Lu et al [18] have put forward a decomposition regime that includes initial and subsequent compensations in order to lower training costs. The gaze assessment could therefore be carried out effectively with the training samples. Nguyen [16] has used procedure to detect and track eye and to train eye image with Gaussian gaze measurement functions. A user must stabilize their head in front of the camera in the front of the user interface application following the training procedure.

Linear model of adaptive regression infers the look of the eye with fewer training samples. Here the appearance based methods depicts the design on the visual characteristics to represent the look can be circumvented. They use an overall eye image to predict a glance by a classifier as a highdimensional input. The classification requires a huge samples consisting of eye pictures. Also different positions on the screen under the different conditions were chosen for the study $[17,19]$.

The technical requirements for photo resolution have generally been reduced but are also sensitive to head motion, light changes and samples used for training purpose.
The functional modules can instead extract the most outstanding visual features to denote the gaze which produces head movements which are not tolerable in nature. The study of gaze with a head movement that offsets the gaze movement when the head moves is quiet significant in nature [15].

\section{PROPOSED SYSTEM}

The proposed system is based on the finest technology i.e., human computer interaction. This interaction is based on our eye and mouth interaction. The interaction is briefly explained using flow diagram starting with the activation of our project till the deactivation of the project.

The below diagram depicts the entire flow of our project. It can be made into three stages. They are:

$\checkmark \quad$ First stage is to detect mouth and eye. The Euclidean distance is determined using the ratio of the eye and the ratio of the mouth appearance

Second stage is the gaze estimation along with the head movement. The directions that are displayed are right and left.

$\checkmark \quad$ Third and final stage is to perform automatic scrolling. This is done by performing squinting of our eyes. The scrolling operation is performed for up, down, right and left.

Figure 1 depicts the entire flow of our project. All the functionalities in it enables to have good interaction with the source machine. Then deactivation process is done by the same functionality that are used for activation of each stages.

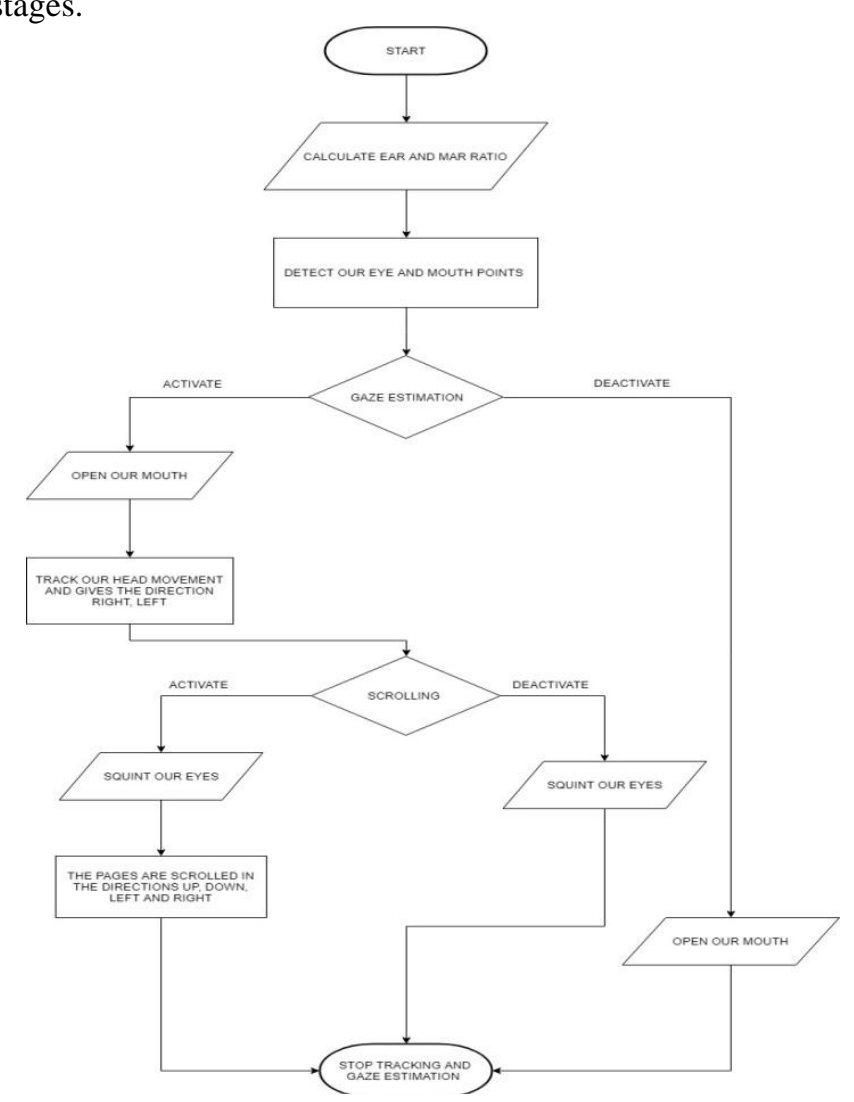

Fig1. Description of workflow of the proposed work

Blue Eyes Intelligence Engineering

\& Sciences Publication 


\section{MODEL DESCRIPTION \& RESULTS}

Machine normally have facial landmarks are trained using the dlib library. The software environment designed for study was built on windows environment. The system designed uses Spyder in anaconda navigator. The analysis is also done using python programming language with necessary packages in it. The results and analysis were done using packages including NumPy library for taking our EAR and MAR points in the array format, pyautogui library is used for the human being detection, imutils library is used for basic image processing that is performed using eye, OpenCV is used for computer vision. The Shape Predictor is the (.dat) format that is accessed only via dlib library file which is in 64-bit encrypted format.

Figure 2 depicts the first stage i.e. detecting the eyes and mouth. Once when the program is executed both eyes and mouth are determined using the EAR and MAR ratio. The he Euclidean Distance is detected in order to determine the points and draw contours.

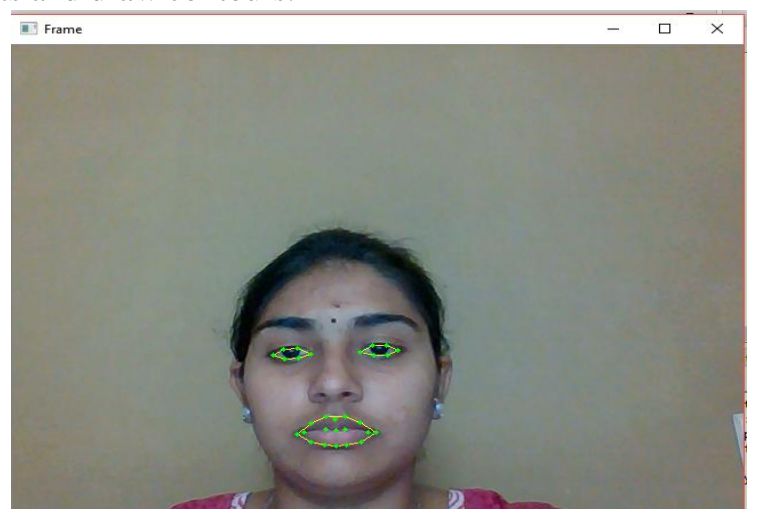

Fig 2. Detecting Eyes and Mouth

Figure 3 depicts the activation procedure of the project i.e. to open our mouth. By performing this functionality, we can enable our system to track our head movement according to the directions.

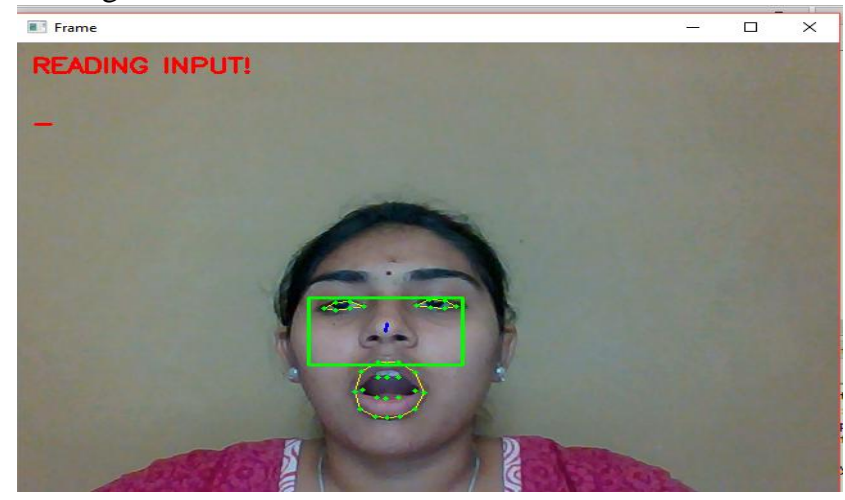

Fig 3: Activation of the Project

Figure 4 depicts the second stage i.e determining the direction according to our head movement. The gaze estimation starts and the mouse cursor point is operated using our head movement. It detects the directions right and left.

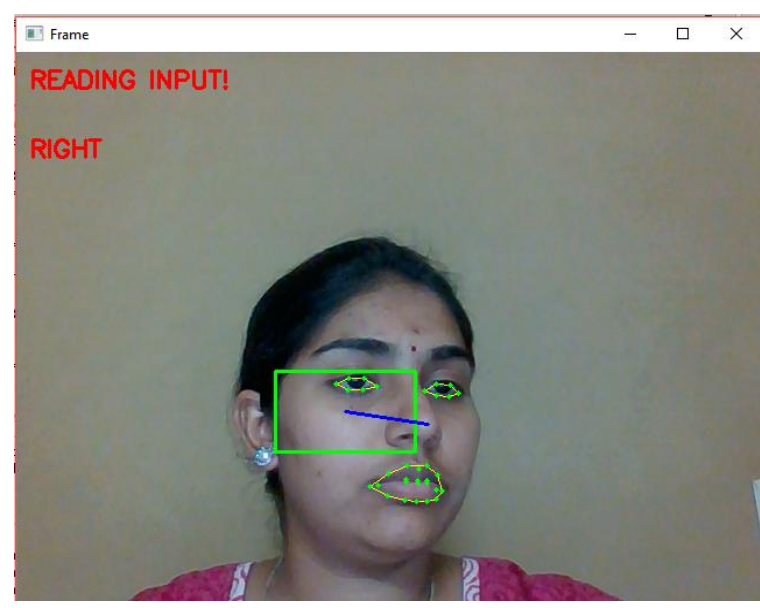

Fig 4. Determination of Direction

Figure 5 depicts the third stage i.e. to perform scrolling by squinting our eyes. Then comes the scrolling operation that is done by squinting our eyes.

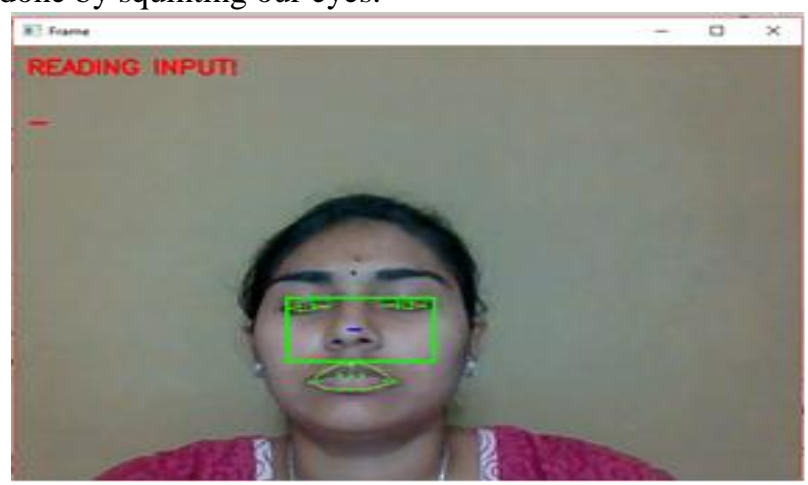

Fig 5. Activation Of Scrolling

Then, there comes the automatic scrolling functionality according to our eye movement. The scrolling of the page automatically occurs in the directions right, left, up and down. Figure 6 depicts the scrolling of the page downwards.

Figure 7 depicts the scrolling of the page upwards.

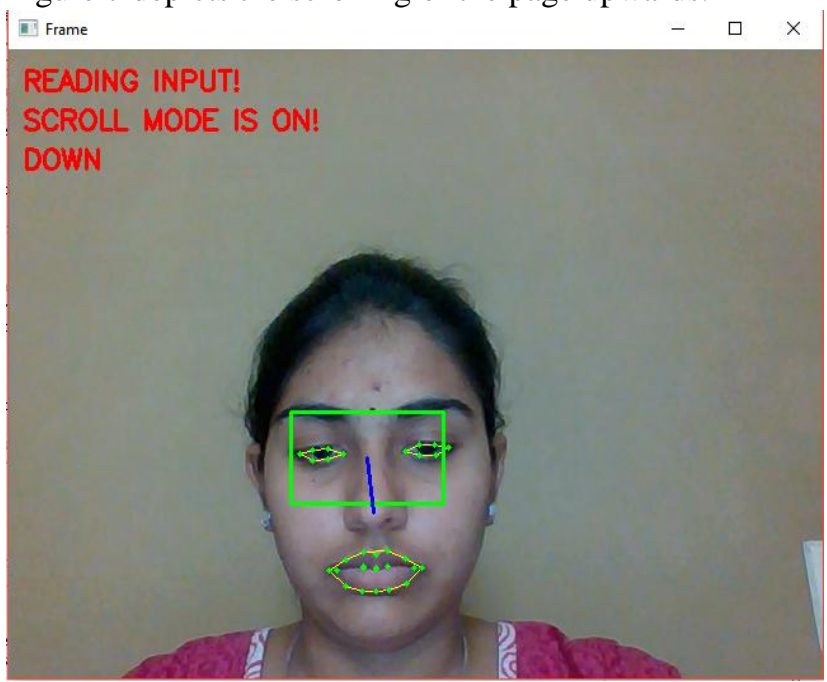

Fig 6. Scrolling Page In Downward Direction

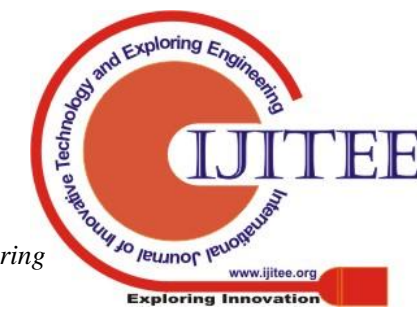




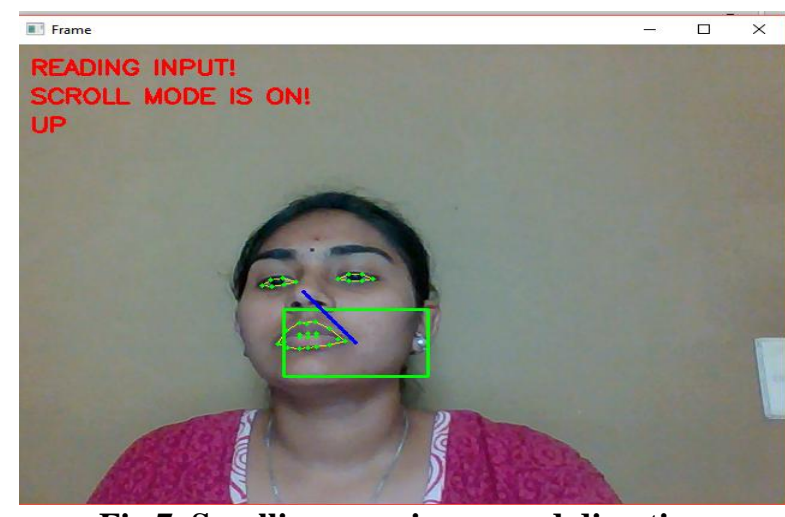

Fig 7. Scrolling page in upward direction

Then at last the deactivation stage comes. The deactivation of the scrolling can be done squinting our eyes. The entire tracking can be deactivated by opening our mouth.

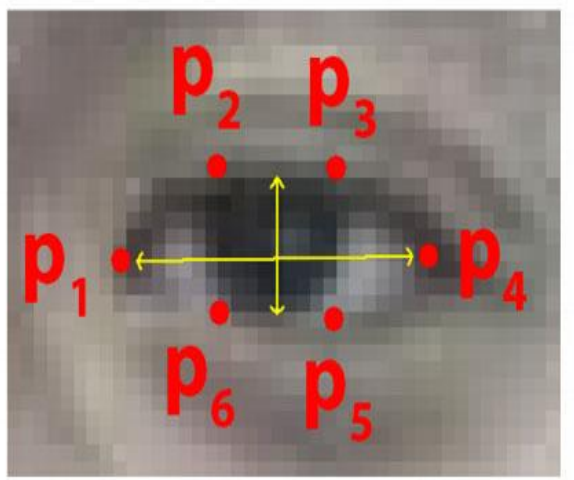

Fig 7. Eye Aspect Ratio

The EAR ratio is used for evaluation which detects the eye movement for performing the expected functionality. The Euclidean Distance can be found using formula given below:

$$
\mathrm{EAR}=\frac{\|p 2-p 6\|+\|p 3-p 5\|}{2\|p 1-p 4\|}
$$

By using the ratio, the eye detection and tracking can be done efficiently. The MAR is used to have the initial activation of the project. By opening or closing of the mouth we can activate or deactivate the eye detection and tracking. Figure 8 depicts the Mouth Aspect Ratio that is also used for determining the Euclidean Distance

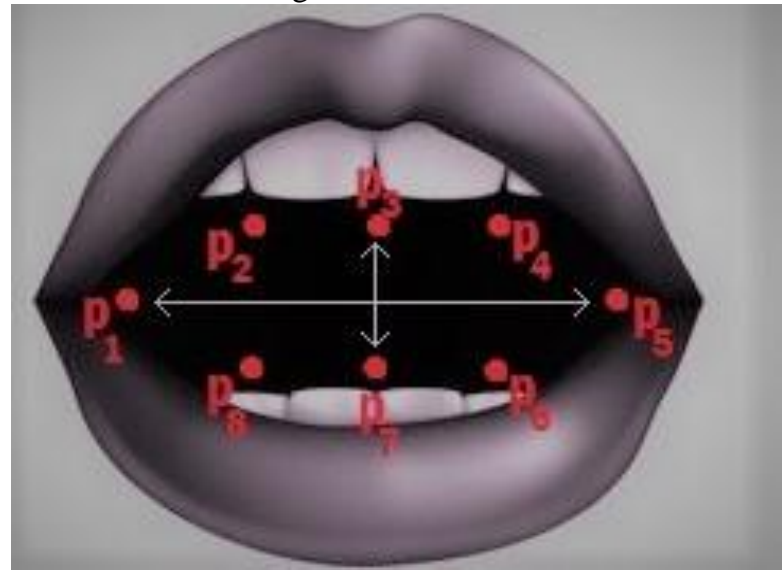

Fig 8. Mouth Aspect Ratio

\section{EVALUATION METRICES}

The evaluation procedure is more important and it is addressed in this section.

\section{a. EYE DETECTION}

The performance measure is used to calculate the accuracy of eye detection that is really a great task. The accuracy generally affects the gaze estimation process with respect to eye centre detection directly. Once the eye is detected, tracking of our eye movement can be done easily.

\section{b. GAZE ESTIMATION}

The gaze estimation is an important factor while dealing with the eye detection. A web-based camera for photo sequences is included in the look-away system. The web camera was installed under a PC monitor and used a resolution of 960 to 720 pixels. Intel Core (TM) i3 CPU $3.40 \mathrm{GHz}$ is a computer hardware configuration. Each subject sat before the computer screen with appropriately 70 $\mathrm{cm}$ distance between the subject and the screen face fully captured by the camera.

\section{CONCLUSION AND FUTURE WORK}

In today's environment, computer plays an efficient role with more interaction among humans. Therefore, some interaction must be made so that we can ease the usage of computer. Our project aim has been achieved to have good interaction using our eye detection and tracking that enables us to have good communication. The future enhancement will be the efficient usage of the interaction in such a way that we can enable us to have advanced working techniques so that interaction can be made in a better way.

\section{REFERENCES}

1 Starner, T., Weaver, J., Pentland, A.: A wearable computer based American sign language recognizer. Assist. Technol. Artif. Intell. 84-96,1998.

2 F. Lu, T. Okabe, Y. Sugano, and Y. Sato, "A head posefree approach for appearance-based gaze estimation," in Proc. Brit. Mach. Vis. Conf., pp. 1-11,2011.

3 Ghaoui, C "Encyclopedia of Human Computer Interaction" Idea Group Reference, 2006.

4 Thoumies, P., Charlier, J.R., Alecki, M., d'Erceville, D., Heurtin, A., Mathe, J.F., Nadeau, G., Wiart, "Clinical and functional evaluation of a gaze controlled system for the severely handicapped Spinal Cord “. 36, 104-109, 1998.

5 Gips, J., DiMattia, P., Curran, F., Olivieri, P. "Using Eagle Eyes an electrodes based device for controlling the computer with your eyes-to help people with special needs". In: Proceedings of the 5th International Conference on Computers Helping People with Special Needs, vol. 1, pp. 77-83, 1996.

6 Kocejko, T., Bujnowski, A., Wtorek, J.: "Eye mouse for disabled. Conference on Human System Interactions", pp. 199-202, 2008

7 Magee, J.J., Scott, M.R., Waber, B.N., Betke, "A realtime vision interface based on gaze detection from a lowgrade video camera. Conference on Computer Vision and Pattern Recognition Workshop”, pp. 159-159, 2004. 
8 Yuille, A.L., Cohen, D.S., Hallinan, P.W "Feature extraction from faces using deformable template" In: Proceedings of IEEE Conference on Computer Vision and Pattern Recognition, pp. 104-109, 1989.

9 Bradski, G., Keahler, A., Pisarevsky, V.: Learning-based computer vision with intel's open source computer vision library. Intel Technol. J. 9(2), 119-130, 2005.

10 J. Zhu and J. Yang, "Subpixel eye gaze tracking," in Proc. 5th IEEE Int. Conf. Autom. Face Gesture Recog., pp. 124-129, 2002.

11 R.Valenti,N.Sebe,andT.Gevers, "Combining head pose and eye location information for gaze estimation," IEEE Trans. Image Process., vol. 21, no. 2, pp. 802-815, 2012.

12 D. Torricelli, S. Conforto, M. Schmid, and T. DAlessio, "A neural-based remote eye gaze tracker under natural head motion," Comput. Methods Programs Biomed., vol. 92, no. 1, pp. 66-78, 2008.

13 I. F. Ince and J. W. Kim, "A 2D eye gaze estimation system with low resolution webcam images," EURASIP J. Adv. Signal Process., vol. no. 1, pp. 1-11, 2011.

14 Y.Sugano,Y.Matsushita,Y.Sato,andH.Koike, "Anincreme ntallearning method for unconstrained gaze estimation," in Proc. Comput. Vis., pp. 656-667, 2008.

15 F. Lu, T. Okabe, Y. Sugano, and Y. Sato, "Learning gaze biases with head motion for head pose-free gaze estimation," Image Vis. Comput., vol. 32, no. 3, pp. 169179, 2014.

16 B. L. Nguyen, "Eye gaze tracking," in Proc. Int. Conf. Comput. Commun. Technol., pp. 1-4, 2009.

17 O.Williams,A.Blake, andR.Cipolla, "Sparseandsemisupervised visual mapping with the S3GP," in Proc. IEEE Int. Conf. Comput. Vis. Pattern Recog.,pp. 230237,2006

18 H.-C. Lu, G.-L. Fang, C. Wang, and Y.-W. Chen, "A novel method for gaze tracking by local pattern model and support vector regressor, "Signal Process., vol. 90, no. 4, pp. 1290-1299, 2010.

19 F. Lu, Y. Sugano, T. Okabe, and Y. Sato, "Adaptive linear regression for appearance-based gaze estimation," IEEE Trans. Pattern Anal. Mach. Intell., vol. 36, no. 10, pp. 2033-2046, Oct. 2014.

\section{AUTHORS PROFILE}

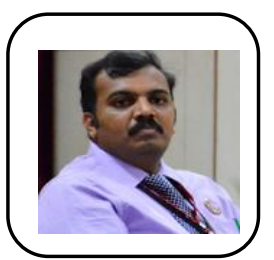

Dr.S.Hariharan holds his undergraduate (B.E), post graduate (M.E) and doctoral (Ph.D) degrees Computer Science and Engineering. Currently he is working as Professor in Department of Computer Science and Engineering, Saveetha Engineering College, India. His research interests includes Information Retrieval, Data mining, Opinion Mining, Web mining. He is a also a member of several associations.He has to his credit several papers in referred journals and conferences. He also serves as editorial board member and as program committee member for several international journals and conferences.

G Nagappan is currently working as Professor in Computer Science

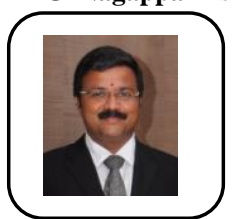
and Engineering department at Saveetha Engineering College, Chennai, India. He received his Ph.D in Information and Communication Engineering from Anna University. He has over 17 years of rich experience in teaching. Additionally he has to his credit, several papers in reputed journals and conferences. His area of specialization includes VANETs,Smart \&Pervasive Computing, Human Computer Interface and Image Processing.

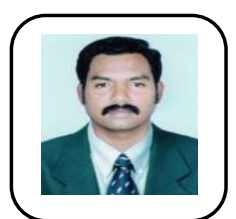

Dr.M.VijayAnand has 22 years of experience in teaching and research, currently working as Professor in the Department of Computer Science and Engineering at Saveetha Engineering College, TamilNadu, India. He completed his Ph.D from Anna University, Chennai. He published more than
30 research papers in International and National Conferences and Journals. His areas of research include Computer Networks, Mobile Ad hoc Networks, Mobile computing and Network Security.

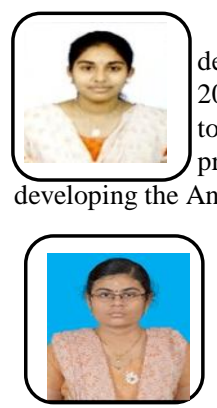

Sree Ranjhani $\mathbf{R}$ S completed her undergraduate degree in Computer Science and Engineering in the year 2019. She was born on November 13. 1997. Her interest is towards Human Computer interaction concepts using the programming Python and also shows interest on Computer Science \& Engineering. She shows her interes
on programming in the languages like C. C++. Python, HTML, JAVA and Android App Development. Currently she is developing a system for smart eye detection and tracking.

Vidhya Sri was born on January 24,1998. She joined the B.E course in 2015 at Saveetha engineering college. Currently pursuing her final year in branch of Computer Science \& Engineering. She shows her interest

(n)

